\title{
Use of Blockchain for Student Record: A Study
}

\author{
Tri A. Sundara ${ }^{1}$, Heru Saputra ${ }^{1}$ and Ilfa Stephane ${ }^{1}$
}

\begin{abstract}
Blockchain, the distributed ledger technology has been expanded beyond its initial conception as a solution for peer-to-peer payment system. It has been used in many areas beyond. This research aims on assessment of Blockchain use case for education field, in particular, student record.
\end{abstract}

Keywords — blockchain, education, student record.

\section{BACKGROUND}

$\mathrm{I}_{\mathrm{s}}^{\mathrm{N}}$ higher education institutions, student records are credentials that always present. It serves as important marker of students' accomplishment, competencies, and other. These records, typically owned by the higher education institution, but there are increasing tendencies that the records would be shared by other institutions as well, such as government and employers. In that condition, reliability and security of these records should be part of the concern.

\section{BLOCKCHAIN}

A blockchain is a distributed database that is used to maintain a continuously growing list of records, called blocks. Each block contains a timestamp and a link to a previous block. A blockchain is typically managed by a peer-to-peer network collectively adhering to a protocol for validating new blocks. By design, blockchains are inherently resistant to modification of the data. Once recorded, the data in any given block cannot be altered retroactively without the alteration of all subsequent blocks and the collusion of the network. Functionally, a blockchain can serve as an open, distributed ledger that can record transactions between two parties efficiently and in a verifiable and permanent way. The ledger itself can also be programmed to trigger transactions automatically. [1] [2] [3]

\section{BLOCKCHAIN AND STUDENT RECORD}

Blockchains are secure by design and are an example of a distributed computing system with high Byzantine fault tolerance. Decentralized consensus has therefore been achieved with a blockchain. This makes blockchains potentially suitable for the recording of events, medical records, and other records management activities, identity management, transaction processing, and documenting

Tri A. Sundara, Lecturer is with Department of Information Systems, STMIK, Indonesia.

Heru Saputra, Department of Information Systems, STMIK, Indonesia. Ilfa Stephane, Department of Information Systems, STMIK, Indonesia. provenance.

Blockchain have these following benefits:

- A robust, truly distributed peer-to-peer system that is tolerant of node failures.

- A network that can identify conflicts and forks and resolve them automatically so as to converge to a single, globally accepted view of events.

- Transparency, verifiability, auditability on the network's activity. We get verifiable processes, whether these concern the exchange and tracking of a digital asset, or a data-driven interaction between parties. Every transaction presents a publicly auditable proof that it was authorized to interact with the system. Eliminates the possibility of disputes, makes reconciliation redundant.

- A method for tagging different pieces of information as belonging to different participants, and enforcing this form of data ownership without a central authority.'

- A system that allows non-trusting participants to interact with each other in a predictable, certain manner. [4]

\section{A. Student Record}

Typical student records include students' data, credits, accomplishment, among others. Some of this data is transactional. These transactional data could benefit from the use of Blockchain.

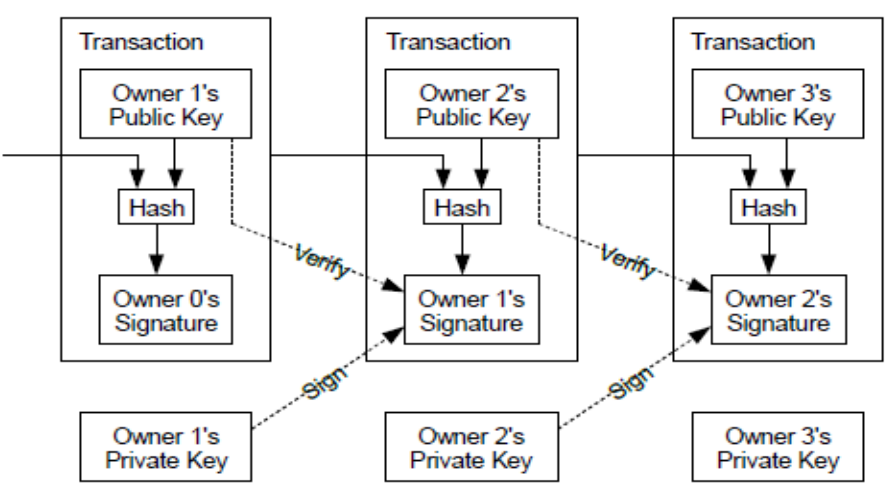

Fig 1. Transaction in Blockchain System

Anyone who needs to verify official records, such as employers, can quickly check the validity and authenticity of each certificate. Any attempt to change, embellish, or otherwise misrepresent a micro-credential represented by a certificate will cause the verification to fail. On the other side, students are increasingly interested in showing the specific skills and abilities they have acquired and developed. By then, fraud detections for online students' record could be leveraged. [5] [6] 


\section{CONCLUSIONS}

It could be concluded that Blockchain has great potential to be used for student record. It may leverage the reliability, security, and ownership of the record. To achieve this purpose, some pilot projects could be conducted.

\section{ACKNOWLEDGMENT}

The authors would like to thank to STMIK Indonesia. This research is funded by 2017 grant from STMIK Indonesia.

\section{REFERENCES}

[1] S. Nakamoto, "Bitcoin : A Peer-to-Peer Electronic Cash System,” pp. 19, 2008.

[2] C. Paper, S. Economy, and P. M. View, "Breaking Down the Blockchain Hype - Towards a Blockchain Market Engineering Approach," no. June, 2017.

[3] M. Swan, Blockchain: Blueprint for a New Economy. 2015.

[4] K. Christidis and G. S. Member, "Blockchains and Smart Contracts for the Internet of Things," vol. 4, 2016.

[5] E. Records, "Educational Records and the Blockchain.".

[6] Y. Cai and D. Zhu, "Fraud detections for online businesses: a perspective from blockchain technology," Financ. Innov., 2016. 